\title{
Emotional Determinants of Sweets Consumption
}

Pilska $\mathrm{M}^{* 1}$ and Nesterowicz $\mathrm{J}^{2}$

${ }^{1}$ Department of Human Nutrition \& Consumption, Warsaw University of Life Sciences, Poland

${ }^{2}$ University of London College Hospital, London, UK

*Corresponding author: Pilska M, Department of Human Nutrition \& Consumption, Warsaw University of Life Sciences, Poland, Tel: +48 225937 145, E-mail: magdalena_pilska@sggw.pl

Citation: Pilska M, Nesterowicz J (2016) Emotional Determinants of Sweets Consumption. J Nutr Health Sci 3(4): 405. doi: 10.15744/2393-9060.3.405

Received Date: September 26, 2016 Accepted Date: November 21, 2016 Published Date: November 23, 2016

\begin{abstract}
The purpose of this study was to investigate the interaction between emotional states and sweets' ingestion, that is, the effect of affective states on sweet snacks' consumption, and the influence of the intake of such products on mood changes. Study participant were 110 students of Warsaw University of Life Science. Data used for the study was obtained through the self-report questionnaire including UMACL mood scale. The study showed that both positive and negative emotions influenced subjects' willingness to consume sweets to the greater level than the sweets' intake itself, though the more salient factor in mood alteration seem to be negative mood. Moreover negative feelings caused subjects' preferences towards sweets rather than healthier snacks. Sweets' ingestion may affect mood and emotions experienced during and after the consumption, depending on feelings of the subjects prior to sweets' intake. These mutual relations may justify the existence of the general opinion that sweets may be consumed to obtain or sustain good feelings, although their influence on emotional state is only temporary.
\end{abstract}

Keywords: Emotions; Mood states; Sweets; Consumption; Craving

\section{Introduction}

\section{Hedonic role of food}

Some studies have examined the existence of emotional eating, and overeating in response to specific mood states. As a result it's now evident that food serves us not only to satisfy our physiological needs but also plays a hedonic role [1]. Hence in this case it's emotions and feelings of the subject that cause the need to consume food. Emotional eating is a consumption of food caused by reasons other than hunger, it is usually perceived as eating in response to negative impulses, and is most frequently considered as overeating without specifically defined mood or emotional state [2]. Some suggest that in emotional eaters the stressful impulse is necessary to induce the intake of sweet, fatty, high-calorie food [3]. According to Sánchez-Benito, et al. neither increased intake, nor overweight or obesity are the outcomes of emotion itself, but they are the outcomes of the way an emotion is perceived and interpreted [4]. Frequently the feeling of 'emotional emptiness' may be interpreted as hunger, and the role of food consumed is to refill emotional deficiencies [5].

While many studies were focused on excessive food intake in response to mood alterations, still less attention has been paid to the relation between emotions or mood and sweets' intake and their interplay. To date studies indicate that one's affective states may have an effect on sweets' consumption or the willingness to consume sweet snacks and vice versa, which justifies the existence of a general opinion that ingestion of mentioned products may have an impact on mood and emotional states. This may not only be due to hedonic properties of food, but may also prove that food in fact regulates those states physically, especially since food addiction and it's action on brain has been pointed out progressively more often [6,7].

\section{Mood regulatory effect of food}

The regulatory effect may be due to the properties of food and its ingredients such as carbohydrates and fat or to food's sensory properties [8,9]. Both of these factors can affect the regions of the brain responsible for feelings of pleasure and reward [8]. Mainly carbohydrates are associated with mood improvement and positive emotions after consumption [10]. This may be confirmed by the theory/existence of carbohydrate craving syndrome, defined as appetite and mood disorder through irresistible desire to consume sweet or starchy food in response to negative emotional states [11]. In this case decreased mood is a trigger to consume high-carbohydrate snacks, which are furthermore considered as more rewarding and more tasty than high-protein snacks for those showing symptoms of abovementioned syndrome [12]. Therefore sugar, as a carbohydrate providing sweet taste may be a 
pivotal factor that affects mood. Low level of blood sugar may cause tendencies to depressive mood, while high blood sugar level may be responsible for mood improvement. Also fatty foods are considered to be in demand when in need to improve emotional state, and they can cause decreased concentration and feeling of calmness [13]. Emotional eating may then be linked with sweetfatty products consumption such as sweet snacks and pastries [14]. However the presumed mood improvement after a meal may also be caused by feeling of satiety, and not by actual mood change [8].

\section{Emotional eating}

This influence of food on alterations in mood and emotional states can be used by individuals to reduce negative feelings as well as to improve or sustain good feelings and emotions. Negative feelings in particular make us reach for sweet snacks, that are the easiest and fastest way to reduce psychological discomfort or provide some sort of distraction. These mood states brought most of the researchers to conclude that mainly negative emotional states are the trigger of emotional eating. However, there's evidence that positive emotions may also affect eating patterns even to the same level as negative emotions. Nonetheless, most of the evidence to date show that increased intake as an outcome of positive mood occurs primarily in individuals with loss of control over eating observed predominantly in obese subjects [15-17]. It's usually suggested, that while experiencing positive mood, food preferred is healthy or salty and fatty food whereas while experiencing negative mood, food preferred is junk food, products of low nutritional value or sweet snacks $[8,18]$. These and many other findings partly lead to conclusions, that sweet as well as fat-dense food and excessive amounts of food are mainly consumed when in negative affective states, and that negative mood induction limits the ability to restrain from food intake in comparison to positive mood $[1,3,19,20]$.

\section{Stress and eating}

While discussing negative emotional states its worth to mention stress. Natural/instinctive reaction to stress is restrained consumption (the higher the stress the lower the consumption). Originally this restraint was to avoid food related sickness or to maintain energy level in unfavourable conditions. Nowadays this reaction is changed due to progressively safer environment, and the tendency for compulsive/binge eating while experiencing stress is frequently observed [1]. Binge eating is defined as repeated episodes during which in short periods of time, usually $2 \mathrm{~h}$, the amount of food consumed is significantly bigger that usually. Feeling of loss of control over eating often accompanies the episodes as well as eating until the feeling of unpleasant fullness, eating excessive amounts of food without hunger, eating faster than usually, eating in isolation, feeling of disgust, guilt or shame after the episodes [21,22]. Compulsive eaters consume usually more energy than healthy individuals, among them women exposed to negative emotions eat more of palatable food and chocolate [23]. The explanation for binge eating episodes in response to negative mood may be increased sensitivity to food related signals appearing in the regions of the brain responsible for feelings of pleasure and reward [24]. Usually the products craved are fat and sugar dense, high carbohydrate foods. Individuals who prefer sweets while binge eating, show tendencies to suffer from those episodes more frequently [22].

Stress may not only be the cause of consumption in order to improve mood, but may also interfere with satiety signalling transmitted to the brain, and as a result may cause loss of control over eating. Loss of control is defined as subjective experience, in which an individual is unable to stop eating or to control the amount or type of food eaten. It is predominant in young overweight people [25]. During the episodes of loss of control the individuals experience inability to control eating behaviours, but the amount of food consumed is ambiguously bigger.

In stressful situations, weakened sensitivity to rewarding properties of food occurs which may lead to food preferences towards sweet and fatty products such as cookies, bread, crisps or frozen products, to snacking more often, consuming more energy, carbohydrates and sodium $[8,26]$. While experiencing stress, high-protein meals are less rewarding than high-carbohydrate meals for subjects with strong loss of control and tendency to consume high-energy food, fatty food, and to decreased consumption of fruits and vegetables is evident $[27,28]$.

\section{Sweets and mood changes}

The impact of sweet snacks on mood changes largely depends on individual traits and susceptibility to different emotions, such as anxiety [9]. Especially adolescents are exposed to emotional eating due to stress associated with own appearance and social contacts with peers [26]. Negative mood induction in adolescents may cause increased intake of carbohydrates, snacks and desserts, and negative feelings may be depleted after such meal [23]. However food ingestion may cause an adverse outcome in stressful or depressive situations. Especially in obese individuals negative feelings may be increased after a meal due to the feelings of guilt and anger caused by overeating [29]. It seems that the factor necessary to induce emotional changes after a meal is sensitivity to their influence or the opposite, insufficient sensitivity to rewarding properties of food. It is especially significant in obese and overweight individuals, who show greater need of consumption in response to negative emotions than slim individuals [8,19,30]. This may be due to the fact that obese individuals are more susceptible to food sensory cues such as smell or visual impulses, as well as to internal cues like anxiety, boredom, fear or depression [5]. In obese women adaptation to pleasure from sweet taste may occur slower that in slim women, what may lead to delayed feeling of satiety, prolonged phase of consumption, and as a result to overeating/weight gain [31]. Moreover women seem to be more vulnerable to mood changes and stress, and they most frequently choose sweets such as ice cream, chocolate and cakes to reduce its level while men choose savoury snacks, like pizza, meat [4,18]. 
Contrarily other study shows that women were more prone than men to limit their consumption in highly stressful situations [5]. Also individuals who consume a large proportion of their daily calories as carbohydrates demonstrate higher sensitivity to their effects on mood, greater mood swings and changes of mental efficacy after carbohydrates' intake. Obese subjects consuming the majority of their daily calories in form of carbohydrates, seem to experience decrease of depressive mood after high-carbohydrate meal, whereas individuals whose diet does not consist of such amounts of carbohydrates show increased drowsiness, tiredness, depression and anxiety after such meal [32]. The pleasure after sweets' consumption is clearly visible especially in overweight or dieting individuals [1]. Obese individuals and the ones reporting carbohydrates craving report mood improvement after a high- carbohydrate meal mood changes such as mental activity rise, manifested by restless, provocative mood resentment or disappointment in contrast to lean individuals or the ones not craving carbohydrates, who show increased drowsiness after such meal or decreased efficacy while cognitive tasks loss of energy or depressive mood [32,33].

\section{Chocolate and mood states}

Food type frequently mentioned as highly rewarding and mood improving is chocolate. Among individuals frequently consuming and craving chocolate, negative mood and emotional eating occurs very often. Aforesaid individuals experience increased arousal, happiness and stronger caving in comparison to individuals eating chocolate occasionally and not craving it [8]. Interestingly, some studies showed that apples as well as chocolate caused increased energy level and mood improvement, but eating chocolate caused additionally feeling of happiness but also feeling of guilt in women [34,35].

Whatever the reason, the regulatory effect of sweets' intake is only temporary and may even be limited only to the consumption phase $[8,34]$. Brief mood improvement is followed by the decline of emotional state, emotional displeasure and reversion to the baseline, negative feelings or feeling of guilt and may cause further consumption $[8,34,36]$.

These relations may cause the development of detrimental eating behaviours, eating disorders or psychological disorders related to inability to solve problems or deal with mood changes. The effect of food on emotional states may contribute to choosing sweets to replace emotional needs that have not been met, and may lead to weight gain and health problems. Therefore it is essential to investigate and explain the presumed causes of disordered eating patterns to improve dietary as well as psychological interventions, and to initiate and incorporate new strategies to manage stress and emotional displeasure.

Therefore the purpose of this study was to examine the mutual influence of emotions and mood, and willingness to consume as well as consumption of sweets, and the relation between baseline emotional states and feelings during and after sweets' intake. We then hypothesized that:

1) the negative mood would be the main trigger inducing craving and consumption of sweets,

2) consumption of sweets would induce mood improvement during and after a meal,

3) while experiencing negative mood the willingness to consume further portions would occur soon after the meal.

\section{Materials and Methods}

The main purpose of the study was to examine the interaction of emotional states, and desire to consume sweets as well as their ingestion. We examined the effect of different emotions and moods on eating behaviours regarding sweet snacks, and the alterations of feelings of the subjects occurring during and after a meal consumed as an outcome of originally experienced affective states.

The data for the study was collected through a self-report questionnaire investigating the relationship between emotional states and sweets' consumption. To rate the mood of the participants the Polish adaptation of the UMACL/UWIST mood adjective scale was used [37]. The UMACL mood questionnaire does not require large samples to show interaction between mood and eating behaviours. The test conducted, measures mood understood as affective experience of moderate duration (at least several minutes), non object-related or quasi object- related, in three dimensions of the core affect: Tense Arousal, Energetic Arousal, and Hedonic Tone. UMACL scale consists of a sheet of 29 printed adjectives. The respondent filling out the sheet was instructed to rate the applicability of each adjective to her/his present mood on a four-point bipolar scale, choosing between the answers 'definitely yes', 'rather yes', 'rather not', 'definitely not' [38].

Each answer was ascribed with a numerical value ranging from 1 to 4 [4 - definitely yes, 3 - rather yes, 2 - rather not, 1 - definitely not, or inversely (R)] (Table 1). The result, being the total of the values/points from the answers vary depending on the scale and may be:

a. for Hedonic Tone 10-40 points

b. for Tense Arousal 9-36 points

c. for Energetic Arousal 10-40 points

Adjectives for each scale and their scoring/values are as follows: 


\begin{tabular}{|c|c|c|c|c|c|c|c|c|c|}
\hline & sweet snacks & salty snacks & dinner plates & fruits & vegetables & diary & bread & fast food & none \\
\hline low HT & 29 & 2 & 12 & 17 & 7 & 5 & 0 & 15 & 12 \\
\hline moderate HT & 24 & 6 & 12 & 39 & 0 & 6 & 0 & 0 & 12 \\
\hline high HT & 18 & 5 & 18 & 11 & 8 & 3 & 5 & 5 & 26 \\
\hline low TA & 14 & 5 & 21 & 16 & 5 & 7 & 5 & 2 & 26 \\
\hline moderate TA & 36 & 3 & 9 & 30 & 3 & 6 & 0 & 3 & 9 \\
\hline high TA & 19 & 8 & 11 & 22 & 8 & 0 & 0 & 17 & 14 \\
\hline low EA & 27 & 6 & 13 & 23 & 6 & 2 & 0 & 8 & 16 \\
\hline moderate EA & 27 & 0 & 15 & 12 & 4 & 12 & 4 & 4 & 23 \\
\hline high EA & 11 & 0 & 21 & 26 & 5 & 5 & 5 & 11 & 16 \\
\hline
\end{tabular}

HT - Hedonic Tone TA - Tense Arousal EA - Energetic Arousal

Table 1: The influence of mood on various food consumption (percentage of respondents given)

Hedonic tone (HT) is defined as subjective feeling of pleasure and is a component of mood describing it in a dimension of pleasure-displeasure. Tense arousal (TA) defines mood in a dimension of tensed-relaxed. Energetic arousal (EA) defines mood in a dimension of energetic-tired, and is also defined as energy to action.

A sten score indicates an individual's approximate position (as a range of values) with respect to the population of values and, therefore, to other people in that population. The individual sten scores are defined by reference to a standard normal distribution. Depending on the score for each scale, sten score (low, medium or high) was designated for each result. Higher numerical value indicates higher level of each mood (Table 2). Levels of each mood depending on sten score are as follows:

a. low scores: sten 1-4

b. medium scores: sten 5-6

c. high scores: sten $7-10$

\begin{tabular}{|c|c|c|c|c|c|c|c|c|c|c|}
\hline & $\begin{array}{c}\text { I eat as } \\
\text { usual }\end{array}$ & $\begin{array}{c}\text { I eat what } \\
\text { tastes me }\end{array}$ & $\begin{array}{c}\text { I don't } \\
\text { feel like } \\
\text { eating }\end{array}$ & $\begin{array}{c}\text { I eat faster } \\
\text { than usual }\end{array}$ & $\begin{array}{c}\text { I eat } \\
\text { slower } \\
\text { than usual }\end{array}$ & $\begin{array}{c}\text { I eat products } \\
\text { another than } \\
\text { usual }\end{array}$ & $\begin{array}{c}\text { I eat more } \\
\text { than } \\
\text { usual }\end{array}$ & $\begin{array}{c}\text { I eat more } \\
\text { than } \\
\text { usual }\end{array}$ & $\begin{array}{c}\text { I don't care } \\
\text { about what } \\
\text { I eat }\end{array}$ & $\begin{array}{c}\text { I think } \\
\text { about food } \\
\text { more often }\end{array}$ \\
\hline JOY & $19,8 \%$ & $36,0 \%$ & $2,5 \%$ & $7,1 \%$ & $6,6 \%$ & $7,1 \%$ & $5,6 \%$ & $6,6 \%$ & $4,6 \%$ & $4,1 \%$ \\
\hline SADNESS & $7,1 \%$ & $16,5 \%$ & $14,2 \%$ & $5,7 \%$ & $12,7 \%$ & $4,7 \%$ & $12,7 \%$ & $13,2 \%$ & $8,5 \%$ & $4,7 \%$ \\
\hline PEACE & $44,0 \%$ & $23,9 \%$ & $1,3 \%$ & $1,3 \%$ & $8,8 \%$ & $4,4 \%$ & $4,4 \%$ & $4,4 \%$ & $3,1 \%$ & $4,4 \%$ \\
\hline STRESS & $4,5 \%$ & $10,5 \%$ & $16,8 \%$ & $15,0 \%$ & $3,2 \%$ & $6,4 \%$ & $12,3 \%$ & $16,8 \%$ & $7,3 \%$ & $7,3 \%$ \\
\hline ANXIETY & $11,5 \%$ & $10,4 \%$ & $19,8 \%$ & $9,9 \%$ & $4,9 \%$ & $4,4 \%$ & $8,2 \%$ & $17,6 \%$ & $8,2 \%$ & $4,9 \%$ \\
\hline BOREDOM & $12,0 \%$ & $17,2 \%$ & $1,7 \%$ & $2,6 \%$ & $6,9 \%$ & $10,3 \%$ & $21,5 \%$ & $0,9 \%$ & $6,4 \%$ & $20,6 \%$ \\
\hline ANGER & $14,3 \%$ & $13,1 \%$ & $22,0 \%$ & $8,9 \%$ & $0,0 \%$ & $3,6 \%$ & $10,1 \%$ & $14,3 \%$ & $9,5 \%$ & $4,2 \%$ \\
\hline
\end{tabular}

Table 2: The influence of selected emotions on various eating behaviors (percentage of respondents given)

Evaluation of mood between positive, neutral an negative was based on relation/ratio between the levels of the three mood dimensions: HT, TA, EA. To classify mood between positive, negative or neutral, the existence of the relations listed below is required:

a. positive mood: high HT, low TA, moderate high EA

b. negative mood: low HT, high TA, low EA

c. neutral mood: moderate HT, moderate/low TA, moderate EA

It is essential to emphasise that based on the relations presented in UMACL scale, we succeed to unambiguously define mood in only $54,5 \%$ of the sample examined, hence all the analysis regarding the relation between mood described as positive, negative or neutral and food choice pertains to this particular share of the sample, in contrast to the results referring to mood dimensions that involve the total of 110 of the participants. The rest of the sample $(45,5 \%)$ has the variation of the moods mentioned above, so it was impossible to define the precise mood state.

Based on the results obtained from the UMACL scale, we analyzed the influence of mood and its three dimensions/components on sweets' craving and consumption.

Food preferences between sweet or healthy snack depending on mood was assessed by choice task with answers being apple or chocolate. Emotions that evoke specific food consumption and following emotional changes were evaluated based on the choice between happiness, sadness, anger, stress, anxiety, calmness, boredom. Feelings accompanying and following consumption of sweet snacks were assessed by the respondents' choice between happiness, sadness, anger, relief, boost of energy, disappointment, guilt. Desire for further consumption was evaluated on the basis of time periods after which the urge to eat occurred, that were 
divided into intervals: none (no desire to eat), right after a meal, 15-30 minutes, 1-3 hours, more than few hours. Desire to consume sweets depending on emotional states between happiness, sadness, anger, stress, anxiety, boredom and calmness was assessed by answering categories being: no difference, more frequently than usually, less frequently than usually. The effect of abovementioned emotions on eating behaviours was assessed by how much the participants endorsed following statements: I eat as usual, I eat what I consider to be tasty, I don't want to eat anything, I eat faster than usually, I eat slower than usually, I eat items I usually don't eat, I eat more, I eat less than usually, I don't pay attention to what I eat, I think of food more often. The willingness to consume and consumption itself were evaluated by time intervals: few times a day, once a day, few times a week, few times a month, once a month, less than once a month, not at all. Body weight was evaluated through $\mathrm{BMI}$ rate. $\mathrm{BMI}<18,5$ was considered as underweight, BMI 18,5-24,9 was considered as normal weight, BMI 25-30 was considered as overweight, BMI $>30$ was considered as obesity. The participants were also asked to evaluate their body weight subjectively between, answering between: too little, normal weight, too much, and also to estimate their body satisfaction and choose between: I would like it to be less, I don't want to change it, I would like it to be more.

Food restriction among the respondents was assessed through the question concerning dieting. From the subjects, individuals who used to diet were selected, and we examined the influence of food restrictions on feelings during and after consumption of sweets. Regularity of meals was assessed by asking 'Do you eat regularly?', with responses being 'yes' or 'no', and by time intervals between meals, which were further divided into time periods: 1-2h, 3-4h, 5 and more hours. Education of the participants was estimated through subjects' response between primary, secondary, higher. Similarly work evaluation was based on the answers: I work full time, I work part time, I don't work. The place of living was assessed through the answers: family home, rented flat, student dormitory, other; and financial status through answering categories ranging between good and bad.

Analysis of the relationship between the emotions experienced and sweets' consumption or willingness to consume sweets, as well as relation between consumption and post-meal feelings and sensations were based upon self-report questionnaire, that is, we entirely depended on subjective appraisal of the subjects of those factors. Based on the results, the statistical analysis of the data obtained was conducted and showed as percentages, ratios, values using data analysis.

\section{Results}

The sample consisted of 110 (94 women, 16 men) students of Warsaw University of Life Sciences. The study was conducted between May and June 2014, and self-report questionnaires were collected.

Mean age of the participants was 23 years old (range 19-28), mean BMI 22,07 (range 17,26-32,65).

$75 \%$ of the participants were normal weight, $12 \%$ and $4 \%$ were overweight and obese respectively.

$9 \%$ of the sample was underweight.

Regularity in meals consumption was found in 53\% of the subjects, time between meals was $3-4$ h for $79 \%$ of the participants, 5 and more hours for $17 \%$, and $1-2 \mathrm{~h}$ for $4 \%$ of the sample.

Higher education and secondary education was declared by $66 \%$ and $34 \%$ of the sample respectively. Full time job had $43 \%$ of the participants, part time job $22 \%$, and unemployed was $35 \%$ of the sample.

Of the participants, $46 \%$ was living at family home, 35\% was renting a flat, $10 \%$ was living in a student dormitory and $9 \%$ chose an option "other".

The financial status was mainly neither good nor bad 30\%. The rest of the respondents were divided between the answers: rather good $29 \%$, good $25 \%$, very good $7 \%$, rather bad $6 \%$, and bad $2 \%$.

\section{The effect of mood on food choice}

Mood of the sample was evaluated on the basis of UMACL scale, describing it as positive, negative or neutral, depending on the relation between the three mood dimensions: hedonic tone (HT), tense arousal (TA) and energetic arousal (EA). The results for these dimensions were as follows. Low HT was observed in 37\% of the participants, moderate HT in 30\%, high HT in 33\%. Low TA was observed in 37\%, moderate TA in $29 \%$ and high TA in 34\% of the participants, and the results for EA were $60 \%$ for low, $24 \%$ for moderate, and $16 \%$ for high EA.

In the choice task, the majority of the participants who chose chocolate as a snack craved during the test was experiencing negative mood (56\%), the minority was experiencing neutral mood (16\%), whereas an apple was chosen most often while experiencing positive mood (43\%), and least frequently while experiencing neutral mood (26\%). Willingness to consume sweets was most frequently associated with negative mood (54\%), less frequently with positive or neutral mood (23\% for each). When analyzing mood dimensions separately, the results were as follows.

For low HT 54\% of the respondents chose chocolate instead of apple (46\%). For moderate HT and high HT apple was chosen more often ( $70 \%$ and $64 \%$ respectively) than chocolate. Along with the increase of HT from low to moderate, also increased the number 
of subjects choosing apple (29\% and 35\% respectively). This relation does not occur in case of chocolate, where the major part of the subjects chose this item for low HT (49\%), then for high and moderate HT ( $29 \%$ and 22\% respectively). Low TA was associated with more frequent choice of apple $(67 \%)$ than chocolate $(33 \%)$, similarly for high TA, apple was chosen by $58 \%$ and chocolate by $42 \%$ of the participants. Conversely, moderate TA was mostly associated with preference of chocolate (52\%) over an apple (48\%). No linear changes in the preference of the subjects were observed for changing intensity of TA either in case of chocolate or an apple. Chocolate was chosen by the majority for moderate (36\%), then for high and low TA (33\% and 31\% respectively), whereas apple was chosen most often for low TA (45\%), least often for moderate TA (23\%). For EA apple was chosen the most frequently, irrespective of intensity of the arousal (low EA 53\%, moderate EA 72\%, high EA 63\% of the participants).

For EA from low to moderate, higher intensity of the arousal was correlated with lower preference of chocolate, then from moderate to high EA it was constant. The majority of the subjects chose chocolate for low EA (68\%), for moderate and high EA it was $16 \%$ each. Along with the increase of EA frequency of choosing apple decreased, for low EA it was $54 \%$, for moderate EA $28 \%$ and for high EA $18 \%$ of the participants.

Sweet snacks were chosen most often (comparing to other food groups) for low HT, moderate TA, and low as well as moderate EA. For low and moderate HT 29\% and 24\% chose sweets respectively, for moderate TA 36\% showed willingness to consume sweets, and for low and moderate EA it was $27 \%$ of the participants each. For high TA the respondents most often reported the willingness to consume fruits (22\%) and sweets (19\%), similarly for low EA (27\% and 23\% respectively) (Table 1).

The study shows that desire to consume sweets decreases with increasing HT. For low HT 44\% of the subjects showed willingness to consume sweet snacks, for moderate HT 30\%, and for high HT 26\% of the sample. This desire/craving changes independently of the intensity of TA (for low TA it's the lowest (24\%), then for high TA (28\%) and for moderate TA (48\%) and decreases with increasing EA. The highest willingness was observed for low EA (64\%), then for moderate (27\%), and for high EA (8\%).

\section{The effect of emotional states on consumption and desire to consume sweets}

In this study the majority of the sample was reaching for sweets while experiencing boredom (29\%), sadness and stress (18\% each), and happiness $(17 \%)$, whereas the minority when angry (4\%). While experiencing calmness or anger the least of the participants reached for sweet snacks.

For all of the emotional states examined, the majority of the subjects reported no changes in willingness to consume sweets. The least of the participants showed alterations in this desire while experiencing calmness, then anger and happiness. No changes in the craving for sweet snacks were observed for anger in $58 \%$ of the participants, for calmness in $77 \%$ of the participants. However, among those who showed such changes, decreased willingness to consume sweets was declared for those emotional states by $28 \%$ and $12 \%$ respectively, while increased willingness to consume sweets was reported by $14 \%$ and $10 \%$ of the sample respectively. While experiencing sadness, stress and boredom increased craving for sweets was frequently observed (43\%, 32\%, $47 \%$ respectively), similar results were obtained for unchanged craving ( $46 \%$ for sadness, $48 \%$ for boredom), whereas decreased willingness to consume sweets was the least frequently observed for those emotional states (12\%, 23\%, 5\% respectively. The shift in this desire as an outcome of experiencing different emotions may also be shown by evaluating the frequency of the sweets' craving appearance/manifestation. While experiencing happiness the major part of the subjects (29\%) showed the desire to consume sweets once a day, whereas while experiencing sadness the major part (37,5\%) declared the desire to consume sweets few times a day or few times a week. When calm the subjects most frequently wanted to consume sweets a couple of times a month (31\%), whereas when experiencing stress $(33 \%)$ or boredom $(50 \%)$ the participants most often declared this desire few times per day. Anxiety was correlated with the willingness to consume sweets few times a week (36\% of the subjects), whereas for anger it was few times per month (43\%). Most frequently the desire to consume sweets occurred when experiencing boredom, sadness and stress.

The results for actual ingestion were slightly different. The study showed that while experiencing happiness as well as sadness the most frequently pointed answer was consumption of sweets few times a week ( $34 \%$ and $50 \%$ respectively). While calm the subjects indicated consumption of sweets less than once a week (29\%), whereas while experiencing stress or anxiety the major part of the sample consumed sweets once a week (29\% and $36 \%$ respectively). Boredom was associated with the intake of sweets few times a day, once a day or less than once a week ( $25 \%$ for each answer). When angry the major part of the sample claimed to consume sweets few times a week or less than once a week (29\%). The most often the participants reported to consume sweets while experiencing boredom and stress.

\section{The effect of sweets' intake on emotions and desire of further consumption}

Of the participants, slightly more than a half was experiencing happiness while eating sweets (51\%), then burst of energy and guilt (17\% and $14 \%$ respectively). Anger and disappointment while eating sweets were experienced by $2 \%$ of the participants each. After the consumption of sweets the majority experienced guilt (33\%), and comparably happiness/contentment (31\%). Significant part of the sample also reported boost of energy (22\%). Some experienced relief (9\%), or disappointment (5\%). There were no feelings of sadness or anger experienced after the sweets' consumption among the participants. 
After the ingestion of sweet snacks the majority of the sample reported no desire to consume further portions (56\%), however $26 \%$ of the sample showed such desire immediately after a snack. $12 \%$ of the group declared willingness to consume next portions of sweets within 15-30 minutes after consumption, $4 \%$ within 1-3h after a meal. The least of the participants showed desire to consume sweets further, after the time period longer than $3 \mathrm{~h}$. The study showed that the longer the time period after consumption, the weaker the desire to consume further portions of sweets.

The study also displays the interaction between the emotional states in which sweets are usually consumed by the participants, and emotions experienced during and after the meal/sweet snacks. When sweets were consumed as a result of feeling happy, the feelings accompanying the intake were happiness (68\%) and boost of energy (16\%), whereas after the meal they were contentment (37\%) or guilt (32\%). Similarly when originally experiencing calmness or boredom, the feelings whilst consumption were for calmness happiness/contentment (60\%) and burst of energy (20\%), and for boredom the same feelings in 59\% and $22 \%$ of the participants respectively. When the subjects were experiencing boredom prior consumption the feelings following the meal were contentment and guilt (34\% and $31 \%$ respectively). When consuming while angry, the feelings accompanying consumption were the most often relieve (50\%), burst of energy (25\%) but also disappointment $(25 \%)$, and an outcome of consuming in this emotional state was feeling of happiness, relief, burst of energy or disappointment experienced by the participants after the consumption. While sad, the volunteers declared experiencing happiness (35\%) or guilt (30\%) during consumption and feelings of guilt (45\%) or happiness (30\%) after the intake. When in stressful situations the subjects likewise showed happiness (40\%) but also relief (20\%), whereas after the consumption they usually experienced guilt (45\%). When the participants were consuming sweet snacks when calm, the most often occurring feeling was burst of energy after the consumption (40\%).

The feelings experienced after the ingestion of sweets were hypothesized to be predictors of further desire to consume following portions. For most of the feelings occurred after sweets' intake, the participants showed no willingness to consume further portions. Among the ones who showed such desire, the majority claimed it to occur immediately after the snack or 15-30mins after. When experiencing happiness after a sweet snack, the participants reported further willingness to eat right after a snack (29\%). Similarly when experiencing boost of energy or guilt after a snack, the desire to further consume sweets appeared forthwith/instantly after a meal (21\% and $31 \%$ respectively). When experiencing relief following the sweets' ingestion most often claimed desire to eat appeared within 15-30 minutes (20\%).

\section{Discussion}

In this study we aimed to show the effect of emotional/affective states on consumption and desire to consume sweet snacks. We also examined the influence of ingestion of such products on the following feelings. Since this mutual interplay has been long studied and suggested to be one of the factors contributing to weight gain and disturbed eating behaviours, we intended to investigate those relations to provide further insight in the plausible impact of mood on sweets' intake and contrariwise.

The influence of affective states on sweet snacks' consumption is evident, and ingestion as a result of experienced emotions is often related to increased intake of sweets [14]. Depending on the type of emotions this consumption may be increased or decreased. Both negative and positive feelings may affect sweets' intake, though negative feelings appear to cause increased ingestion of sweets significantly more often than positive feelings. In this study when negative mood was experienced, sweet snacks were preferred over healthy choices. These findings are parallel with the study of Habhab, et al. and Oliver, et al. who have confirmed that negative mood may be the cause of ingestion of more palatable and fat-dense foods [3,39]. In the present study sadness, stress and boredom seemed to have the most significant impact on increased desire to consume sweets, and the most frequently chosen products improving mood were chocolate and chocolate products. The majority of the sample declared the intake of sweets while experiencing boredom. In this emotional state the subjects also craved sweets more often. This may be the evidence of sweets being perceived as a distraction or a sort of stimulant, since the intake provided boost of energy during and after a meal for a significant part of the sample. The major number of the respondents also showed sweets' consumption when feeling sad or stressed in contrast to feelings that are not related to emotional tension, such as calmness. Also Wansink and SeaBum have claimed that negative mood contributes to sweet snacks' ingestion [18]. Stress is often mentioned to be associated with increased intake of sweet, high-carbohydrate, high-fat and high-calorie food [3,26,40]. Also when in stress a high-protein meal may be less rewarding than a high-carbohydrate meal [27]. Ingestion of sweets in stressful situations may be aimed at bringing consolation and relief, or suppressing negative feelings, since those products are perceived as highly rewarding [41,42]. The results of the current study confirm that sweets' ingestion causes positive feelings in the majority of the sample. However in our study, despite emotional tension that accompanies anger, we did not observe any effect of this emotional state on changes in sweets' intake, though consuming sweet snacks as an outcome of feeling angry lead to subjects experiencing relief during consumption, while the feelings most often following the intake were boost of energy, relief, happiness but also disappointment. Opposite results were provided by the study of Appelhans, et al., showing that the subjects consumed more energy as snacks while feeling angry that in neutral mood [43]. The contradictory results may be due to the fact that in the abovementioned study obese individuals were examined, whereas in the present study the majority of the sample were individuals with normal body weight. This may lead to a conclusion that the relation between emotions and eating behaviours may vary depending on individuals' body weight/BMI. 
Also experiencing positive mood may result in increased intake of sweets and high-calorie food, especially in obese individuals [19]. Contrarily some suggest that positive feelings prior to a meal may encourage to choose healthier snacks, and support the selection of healthier next meal or salty and fat-dense snacks [18,44]. Also Christensen and Pettijohn have proved that negative feelings were associated with junk food consumption, whereas positive feelings were related to consumption of healthy meals [10]. In the current study positive emotions did not affect sweets' consumption as strongly as negative feelings, and positive mood seemed to cause food choice towards healthier snacks. The data obtained from this study shows that this relation was more salient, when the effect of mood on food choice was evaluated by analyzing the three components of mood, HT, TA and EA. Our findings confirmed that experiencing limited pleasure, moderate tension and low energetic arousal causes the preference of sweets over healthy products. The choice task incorporated in the present study corroborated that mood of the subjects affected food preferences. An apple was chosen most often when the subjects were experiencing positive mood, chocolate when they were experiencing negative mood. This is in accordance to the study of Leszczyńska, et al. that has proved that vegetables were preferred when in positive mood, whereas sweet, high-carbohydrate snack were preferred when in negative mood $[45,46]$.

Our study suggest that when the hedonic feelings are lower (low HT), the subjects tend to reach for chocolate possibly to induce good feelings, since it is thought that mainly sweet products provide required pleasure [1]. The results of the current study show that with increased HT from low to moderate increases the number of the subjects choosing apple instead of chocolate. Although numerous findings to date (some of them cited above) confirm the impact of strong emotional tension on increased sweets' consumption this study did not succeed in confirming a relation where the higher the TA the higher the number of the individuals choosing chocolate. Our findings show that only moderate TA contributed to preference of the subjects of chocolate over apple. For EA an apple was chosen more frequently irrespective of its intensity, though for low EA the numbers of participants choosing apple and chocolate were similar, and we observed the tendency where with the increase of EA from low to moderate, decreased the number of the individuals choosing chocolate. Similar relation was observed for apple, where with increased intensity of EA decreased successively the number of participants choosing this item. When in emotional discomfort chocolate may be preferred because it usually is perceived as mood improving, although its consumption in response to negative feelings and its effect on mood are more evident in individuals consuming chocolate regularly [36]. Interestingly Macht and Dettmer have shown that chocolate ingestion as well as an apple ingestion caused mood improvement and boost of energy, but chocolate caused also feelings of happiness and pleasure [34]. However in women the feeling of guilt after the intake of chocolate was also observed. Martin, et al. have confirmed that ingestion of milk chocolate may diminish anxiety, although since in their study dark chocolate and crackers caused increased anxiety, they concluded that mood improvement may be not only due to carbohydrates and fat contained in food, but also due to sensory properties of milk chocolate [9]. This is in accordance to the results of our study, which show that while in negative mood also the desire to consume fruit was increased. The most frequent willingness to consume fruit was observed for moderate HT, moderate and high TA, low and high EA. This may lead to a conclusion that also sweet taste or only carbohydrates without fat may regulate emotional states, and in case of the three mood dimensions sweets' intake is supposed to improve mood when low or moderate feeling of pleasure, or moderate or high emotional tension is experienced, and may induce or maintain energetic arousal.

Based on these studies we may conclude that food consumption depends on emotional state, and ingestion of sweet snacks may act as a moderator of postprandial feelings and is most often associated with maintaining positive mood or improving negative mood. Those alterations largely depend on the feelings experienced prior to the consumption. In our study sweets' intake as a result of different emotions was associated with changes in emotional states during and after a meal. The feeling occurring most frequently while consuming sweets was happiness, whereas after a sweet snack it was guilt, then contentment and burst of energy. The fact that despite good feelings accompanying consumption, following feeling of guilt was often reported, may prove that the regulatory effect of sweets on mood is only temporary. Considering that among the participants who showed further desire to consume sweets most of them declared this urge right after or 15-30 minutes after sweet snack, the hypothesis that sweets are an ineffective way of coping with negative emotions and unwanted feelings is supported. This desire depended on the feelings following consumption. In the study we observed that for positive feelings such as happiness or burst of energy as well as for negative feelings like disappointment or guilt appeared after the intake, if the desire to consume sweets was observed, it was most often immediately after a sweet snack. Negative feelings prior to the consumption of sweets may then be associated with enhanced mood during and declined mood after the intake, and then result in immediate desire to consume further portions of sweet snacks. These results are in line with the study of Hetherington and MacDiarmid, where mood improvement was observed only during the consumption of carbohydrates, but after a meal the negative feelings arose and returned to the baseline level preceding the intake [34]. Those results may reflect an attempt of the subjects to sustain good feelings or improve negative feelings through sweets, or to induce positive feelings experienced during consumption. This is partly in accordance with another study which has shown that food may be consumed to reduce negative feelings, especially in girls (adolescents with loss of control) [23]. Those results however are contradictory to the results of the study of Haedt-Matt and Keel, proving that neither palatable food consumption nor consumption of food in general did cause improvement in mood in adults with loss of control, and that negative emotions increased after compulsive/binge eating episodes [47]. In our study we did not take into consideration the tendency for/ susceptibility to compulsive behaviours as a factor moderating/affecting sweets' intake, hence the results of the studies may vary depending on the propensity of the subjects for loss of control over eating. 
Again, the results of this study suggest that desire to consume sweets may be increased due to negative emotions such as sadness, stress or boredom, though anger or anxiety, that are considered as negative feelings were positively associated with decreased craving/ did not cause mentioned changes. In the state of sadness, stress or boredom the desire to consume sweet snacks occurred more often (even few times a day) than for example while feeling happy (once a day) or calm (few times a month). This relation may be due to those products being perceived as mood improving and as a way to deal with difficult feelings in subjects unable to manage emotional discomfort. Wurtman claims that negative feelings may cause strong craving for sweet and starchy snacks [11]. However, also happiness was associated with slightly increased desire to consume sweets, mainly chocolate products and ice cream, which may indicate the willingness to maintain good feelings. In our findings the willingness to consume sweets did not reflect/translate into actual consumption which is evidently less frequent. The ingestion was most often declared for happiness, sadness and anger (few times a week), and for stress and anxiety (once a week). The most frequent consumption was for boredom and it was even few times a day, however in this state the same number of the subjects claimed consumption once a day and less than once a week. Those differences may be due to avoiding too frequent consumption of sweets, and given that the majority of the sample were women, preoccupation regarding diet and slim figure seems to be the cause of this restriction. Some of the participants declared deliberate restraint from sweets' consumption to maintain a healthy diet or to decrease body weight, therefore the results showing the desire to consume sweet snacks may be divergent from actual intake.

The effects of sweets mentioned above may be ascribed to the components of sweet snacks such as carbohydrates and fat. Fat and simple carbohydrates mainly in palatable food may induce feelings of pleasure during consumption and stimulate positive emotional states and feelings of reward [13]. Moreover, when in negative mood, the effect of mentioned products may appear stronger for the subjects and interfere with signals of satiety, contributing to consumption of palatable food in order to regulate emotional states, even when hunger is not experienced [13]. Also a study of Ranzenhofer, et al. has shown that negative mood caused increased consumption of carbohydrates in general, snacks and deserts [23]. However high carbohydrate food ingestion may cause mood alterations with temporary feeling of relief and following feeling of disappointment, resentment, anger or even aggression. In long-term carbohydrates might cause loss of energy and development as well as continuation of depressive state. Mood improvement may be often limited only to the consumption phase, hence positive feelings accompanying consumption of high-carbohydrate food may be associated with feeling of satiety [8]. Other findings provide a theory that it's restraint in carbohydrate consumption that causes mood improvement, and that their intake is most often associated with feeling of guilt however according to Udo, et al. restraint from consumption may result in increased stress in obese individuals that subsequently weakens the ability of the subjects to refrain from eating high-calorie foods $[19,36]$. According to our data while in negative mood also the desire to consume fruit was increased what may suggest mood improving properties of not only the ingredients of the products such as carbohydrates, but also of sweet taste. Given that the outcomes of different studies are often ambiguous and contradictory, it is possible that the impact of carbohydrates on mood changes is due to individual traits and susceptibility to their action, and high- carbohydrate snacks may improve mood mainly in individuals who consume those macronutrients regularly in considerable amount /which may be a result of the amount/content of the carbohydrates in diet [32].

Our study as well as the cited studies shows that sweets, mainly due to their ingredients and sensory properties may regulate emotional states. Mood modulation through sweet snacks may cause their intake as a way to provide changes in affective/emotional states. Sweets are often a way to adjust emotional states, that is, enhance positive mood or improve negative feelings. Nevertheless ingestion of sweets to obtain mentioned results may indicate the incapability to manage own emotions, may be an attempt to alleviate unpleasant feelings or replace unmet emotional needs or to induce positive feelings when the subject is unable to solve problems. Positive and negative emotions affect sweets' ingestion, as well as sweet snacks' consumption may affect mood and feelings during and after a meal. However this interaction depends on different factors such as diet, body weight, eating behaviours that were not standardized in this study, thus potential influence of those variables on eating patterns such as compulsive eating or loss of control over eating were lacking. The differences in results obtained from our study and other studies may arise from those individual traits of the subjects.

The evaluation of mood based on UMACL scale showed significant relations between experienced emotions and mood, and eating behaviours, although these relations were analyzed on the basis of self-report questionnaires where the subjects evaluated their emotions subjectively, recalling the feelings usually experienced when in situations related to food consumption, which may then be over or under reported. The results largely relied on the subjects' estimation/appraisal of these factors, hence may be biased and influence the outcomes of the study. However this may be a strength to this study, since the findings obtained from laboratory environment may not reflect eating behaviors presented in natural conditions.

Because of those reasons and taking into account the ambiguous results of different analysis and the fact that ingestion of sweets as a way to regulate emotional states may lead to maladaptive eating patterns, eating disorders or weight gain, further investigation in the area of the relationship between emotions and sweet snacks' intake is required.

\section{Conclusions}

- Mood and emotions influence the eating of sweets. Positive and negative emotions change the craving for sweets.

- Additionally sweets consumption clearly regulates emotions and mood changes during and after consumption. 
- Emotional states, positive and negative, determine sweets craving which proves that sweets can improve negative mood and evoke or sustain positive moods. Sadness, stress and boredom have larger potential to evoke sweets craving.

- Chocolate was the sweet product the most frequently chosen that has potential to influence moods and emotional states, especially negative. Modulating influence of sweets on mood is rather short-term.

\section{References}

1. Privitera GJ, Misenheimer M, Murali Doraiswamy P (2013) From weight loss to weight gain. Appetite changes in major depressive disorder as a mirror into brain-environment interactions. Front Psychol 4: 873.

2. Nguyen-Rodriguez ST, Unger JB, Spruijt-Metz D (2009) Psychological Determinants of Emotional Eating in Adolescence. Eat Disord 17: 211-24.

3. Oliver G, Wardle J, Gibson EL (2000) Stress and food choice: a laboratory study. Psychosom Med 62: 853-65.

4. Sánchez Benito JL, Pontes Torrado Y (2012) Influencia de las emociones en la ingesta y control de peso. Nutr Hosp 27: 2148-50.

5. Ogden J (2010) The Psychology of Eating: From Healthy to Disordered Behavior. Wiley-Blacwell.

6. Gearhardt AN, Grilo CM, Dileone RJ, Brownell KD, Potenza MN (2011) Can food be addictive? Public health and policy implications. Addiction 106: $1208-12$.

7. Parylak SL, Koob GF, Zorrilla EP (2011) The dark side of food addiction. Physiol Behav 104: 149-56.

8. Blackman MC, Kvaska CA (2011) Nutrition psychology. Improving dietary adherence. Jones and Bartlett Publishers, Massachusetts.

9. Martin FP, Antille N, Rezzi S, Kochhar S (2012) Everyday Eating Experiences of Chocolate and Non-Chocolate Snacks Impact Postprandial Anxiety, Energy and Emotional States. Nutrients 4: 554-67.

10. Christensen L, Pettijohn L (2001) Mood and carbohydrate cravings. Appetite 36: 137-45.

11. Wurtman JJ (1990) Carbohydrate craving: relationship between mood and disorders of carbohydrate intake. Drugs 39: 49-52.

12. Corsica JA, Spring BJ (2008) Carbohydrate craving: A double-blind, placebo controlled test of the self- medication hypothesis. Eat Behav 9: 447-54.

13. Dye L, Lluch A, Blundell JE (2000) Macronutrients and mental performance. Nutrition 16: 1021-34.

14. de Lauzon B, Romon M, Deschamps V, Lafay L, Borys JM, et al. (2004) The three-factor eating questionnaire-r18 is able to distinguish among different eating patterns in a general population. J Nutr 134: 2372-80.

15. Evers C, Adriaanse M, de Ridder DTD, de Witt Huberts JC (2013) Good mood food. Positive emotion as a neglected trigger for food intake. Appetite 68: 1-7.

16. Yeomans MR, Coughlan E (2009) Mood-induced eating. Interactive effects of restraint and tendency to overeat. Appetite 52: 290-8.

17. Patel KA, Schlundt DG (2001) Impact of moods and social context on eating behavior. Appetite 36: 111-8.

18. Wansink B, Sea Bum P (2000) Accounting for taste. Prototypes that predict preference. J Database Marketing 7: 308-20.

19. Udo T, Grilo CM, Brownell KD, Weinberger AH, DiLeone RJ, et al. (2013) Modeling the Effects of Positive and Negative Mood on the Ability to Resist Eating in Obese and Non-obese Individuals. Eat Behav 14: 40-6.

20. Ludwig DS, Majzoub JA, Al-Zahrani A, Dallal GE, Blanco I, et al. (1999) High glycemic index foods, overeating, and obesity. Pediatrics 103: E26.

21. American Psychiatric Association (2000) Diagnostic and Statistical Manual of Mental Disorders, text revision. American Psychiatric Association, Washington.

22. Avena NM, Rada P, Hoebel BG (2009) Sugar and Fat Bingeing Have Notable Differences in Addictive- like Behavior. J Nutr 139: 623-8.

23. Ranzenhofer LM, Hannallah L, Field SE, Shomaker LB, Stephens M, et al. (2013) Pre-Meal Affective State and Laboratory Test Meal Intake in Adolescent Girls with Loss of Control Eating. Appetite 68: 30-7.

24. Bohon C, Stice E (2012) Negative Affect and Neural Response to Palatable Food Intake in Bulimia Nervosa. Appetite 58: 964-70.

25. Tanofsky-Kraff M (2008) Binge eating among children and adolescents. in: Jelalian E, Steele R (eds.) Handbook of Child and Adolescent Obesity. Springer Publishers, New York

26. Jeong EY, Kim KN (2007) Influence of stress on snack consumption in middle school girls. Nutr Res Pract 1: 349-55.

27. Lemmens SG, Martens EA, Born JN, Martens MJ, Westerterp-Plantenga MS (2011) Lack of effect of high-protein vs. highcarbohydrate meal intake on stressrelated mood and eating behavior. Nutr J 10: 136.

28. Nguyen-Michela ST, Ungera JB, Spruijt-Metza D (2007) Dietary Correlates of Emotional Eating in Adolescence. Appetite 49: 494-9.

29. Popless-Vawter S, Brandau C, Straub J (1998) Triggers of overeating and related intervention strategies for women who weight cycle. Appl Nurs Res 11: 69-76.

30. Cannetti L, Bachar E, Berry EM (2002) Food and Emotion. Behav Processes 60: 157-64.

31. Pepino MY, Mennella JA (2012) Habituation to the Pleasure Elicited by Sweetness in Lean and Obese Women. Appetite 58: 800-5.

32. Lieberman HR, Wurtman JJ, Chew B (1986) Changes in mood after carbohydrate consumption among obese individuals. Am J Clin Nutr 44: 772-8.

33. Ogden J, Wardle J (1991) Cognitive and emotional responses to food. Int J Eat Disord 10: 297-311.

34. Macht M, Dettmer D (2006) Everyday mood and emotions after eating a chocolate bar or an apple. Appetite 46: 332-6.

35. Hetherington MM, MacDiarmid JI (1993) "Chocolate addiction": a preliminary study of its description and its relationship to problem eating. Appetite 21: 233-46.

36. Hill A J, Weaver CF, Blundell JE (1991) Food craving, dietary restraint and mood. Appetite 17: 187-97.

37. Goryńska E, Mathews G, Chamberlain AG, Jones DM (2005) UMACL - Przymiotnikowa Skala Nastroju UMACL. Polish Psychological Association.

38. Mathews G, Chamberlain AG, Jones DM (1990) Refining the measurement of mood: The UWIST Mood Adjective Checklist. Brit J Psychol 81: 17-42.

39. Habhab S, Sheldon JP, Loeb RC (2009) The relationship between stress, dietary restraint, and food preferences in women. Appetite 52: 437-44.

40. Cartwright M, Wardle J, Steggles N, Simon AE, Croker H, et al. (2003) Stress and dietary practices in adolescents. Health Psychol 22: 362-9.

41. Rutters F, Nieuwenhuizen AG, Lemmens SG, Born JM, Westerterp-Plantenga MS (2009) Acute stress- related changes in eating in the absence of hunger. Obesity (Silver Spring) 17: 72-7. 
42. Lemmens SG, Rutters F, Born JM, Westerterp-Plantenga MS (2011) Stress augments food 'wanting' and energy intake in visceral overweight subjects in the absence of hunger. Physiol Behav 103: 157-63.

43. Appelhans BM, Whited MC, Schneider KL, Oleski J, Pagoto SL (2011) Response style and vulnerability to anger-induced eating in obese adults. Eat Behav 12: 9-14.

44. Lu J, Huet C, Dubé L (2011) Emotional reinforcement as a protective factor for healthy eating in home settings. Am J Clin Nutr 94: 254-61.

45. Leszczyńska S, Błażejewska K, Lewandowska-Klafczyńska K, Rycielski P (2011) Emocje a zachowania żywieniowe u kobiet w wieku 18-30 lat. Endokrynologia, Otyłość i Zaburzenia Przemiany Materii 7: 167-71.

46. Martin CK, Rosenbaum D, Han H, Geiselman P, Wyatt H, et al. (2011) Change in food cravings, food preferences, and appetite during a low- carbohydrate and low-fat diet. Obesity (Silver Spring) 19: 1963-70.

47. Haedt-Matt AA, Keel PK (2011) Revisiting the affect regulation model of binge eating: a meta-analysis of studies using ecological momentary assessment. Psychol Bull 137: 660-81.

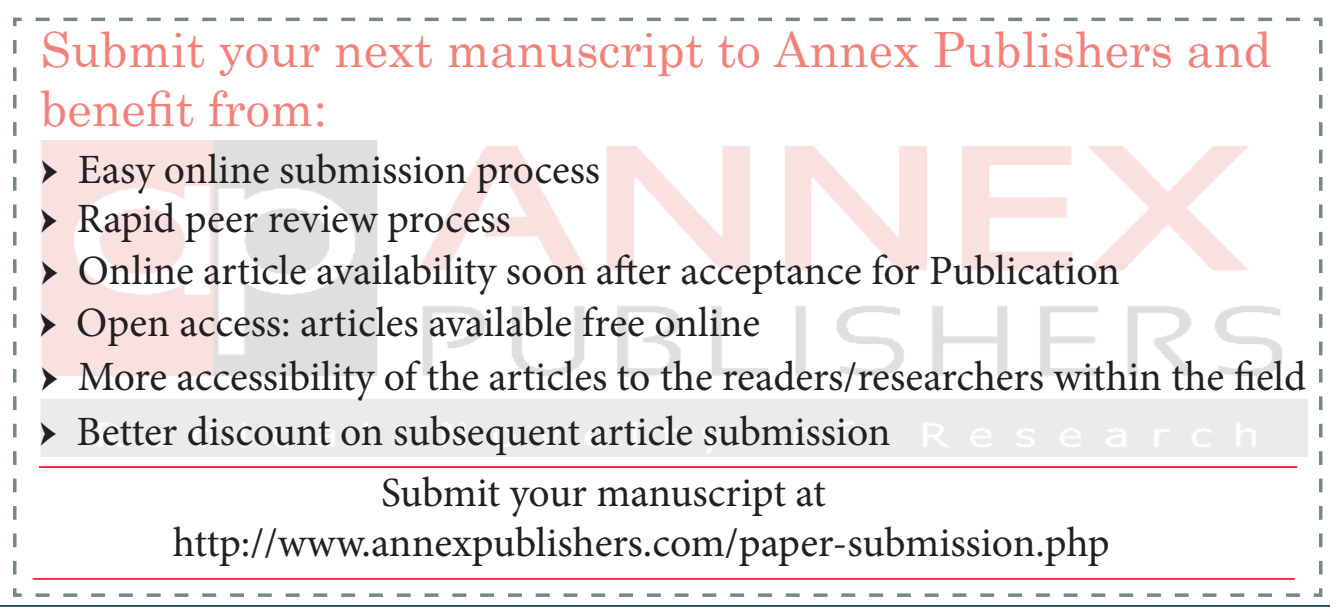

